\title{
Expression of acid-sensing ion channels and selection of reference genes in mouse and naked mole rat
}

\author{
Laura-Nadine Schuhmacher ${ }^{1,2}$ and Ewan St. John Smith ${ }^{1 *}$
}

\begin{abstract}
Acid-sensing ion channels (ASICS) are a family of ion channels comprised of six subunits encoded by four genes and they are expressed throughout the peripheral and central nervous systems. ASICs have been implicated in a wide range of physiological and pathophysiological processes: pain, breathing, synaptic plasticity and excitotoxicity. Unlike mice and humans, naked mole-rats do not perceive acid as a noxious stimulus, even though their sensory neurons express functional ASICs, likely an adaptation to living in a hypercapnic subterranean environment. Previous studies of ASIC expression in the mammalian nervous system have often not examined all subunits, or have failed to adequately quantify expression between tissues; to date there has been no attempt to determine ASIC expression in the central nervous system of the naked mole-rat. Here we perform a geNorm study to identify reliable housekeeping genes in both mouse and naked mole-rat and then use quantitative real-time PCR to estimate the relative amounts of ASIC transcripts in different tissues of both species. We identify RPL13A (ribosomal protein L13A) and CANX (calnexin), and $\beta-A C T I N$ and EIF4A (eukaryotic initiation factor 4a) as being the most stably expressed housekeeping genes in mouse and naked mole-rat, respectively. In both species, ASIC3 was most highly expressed in dorsal root ganglia (DRG), and ASIC1a, ASIC2b and ASIC3 were more highly expressed across all brain regions compared to the other subunits. We also show that ASIC4, a proton-insensitive subunit of relatively unknown function, was highly expressed in all mouse tissues apart from DRG and hippocampus, but was by contrast the lowliest expressed ASIC in all naked mole-rat tissues.
\end{abstract}

Keywords: Acid-sensing ion channel, Gene expression, geNorm, Normalization, Quantitative real-time PCR, Naked mole-rat, Housekeeping gene selection, Central nervous system, Comparative study

\section{Introduction}

Acid-sensing ion channels (ASICs) are a group of six ion channels encoded by four different genes, splice variants of the genes encoding ASIC1 and ASIC2 produce ASIC1a and ASIC1b, and ASIC2a and ASIC2b respectively [1]. The predominant endogenous activator of ASICs is protons and combinations of different ASIC subunits produces homoand heterotrimers with different biophysical and pharmacological properties [2-4]. Thus the sensitivity and response to protons of a particular neuron depends upon the combination of ASICs that are expressed. Presynaptic stimulation has been shown to produce changes in

\footnotetext{
* Correspondence: es336@cam.ac.uk

'Department of Pharmacology, University of Cambridge, Tennis Court Road, Cambridge CB2 1PD, UK

Full list of author information is available at the end of the article
}

extracellular $\mathrm{pH}$ through release of acidic synaptic vesicles into the synaptic cleft and thus protons may act as a neurotransmitters, with ASICla expressed in the postsynaptic membrane contributing to synaptic plasticity [5]. ASICs therefore have the potential to contribute to a variety of physiological and pathophysiological processes in the brain and evidence exists to support their involvement in: addiction [6], anxiety/fear [7-9], regulation of breathing [10], learning and memory [11] and excitotoxicity [12]. In the peripheral nervous system, ASICs are expressed by sensory neurons of the dorsal root ganglia (DRG) [13-16] and are involved in nociception and mechanosensation [17, 18]. Interestingly, the naked mole-rat (Heterocephalus glaber) shows no nocifensive response to acid due to a lack of activation of cutaneous sensory neurons [19], even though DRG neurons have ASIC-like currents and cloned ASICla 
and ASIC1b, as well as TRPV1, have biophysical properties that are indistinguishable from those of mice [14, 20]. Behavioral acid insensitivity is accounted for by a genetic variation in the voltage-gated $\mathrm{Na}^{+}$channel subunit 1.7 (NaV1.7), which confers enhanced acid inhibition [14]. The $\mathrm{NaV} 1.7$ variation is conserved in a variety of hibernating species [21] and insensitivity to acid in these species is likely due to an adaptation to living in a hypercapnic environment $[14,22]$. Considering that NaV1.7 expression is largely restricted to the peripheral nervous system [23, 24], any central adaptations to hypercapnia may result from differential ASIC expression and thus it is necessary to determine if there are any differences in ASIC brain expression in the naked mole-rat compared to mouse.

ASIC expression has been investigated using a variety of methods. ASIC1 and ASIC2 been found to be predominantly expressed in the brain by in situ hybridization, with a high level of mRNA expression in the cerebellum, hippocampus, habenula, amygdala and olfactory bulb [25-27]. This ASIC1 expression pattern was recapitulated by immunohistochemistry and northern blot analysis of human ASIC1 and ASIC2 showed transcripts in the amygdala, hippocampus, thalamus, substantia nigra, subthalamic nuclei and caudate nucleus [7]. Using probes that differentiated between ASICIa and ASIC1b, it has been shown that whereas ASIC1a is expressed in rat DRG, spinal cord and brain, ASIC1b expression is restricted to the DRG [28]. However, reverse-transcriptase PCR (RT-PCR) analysis showed that $A S I C 1 b$, along with all other $A S I C$ transcripts, is expressed in mouse anterior pituitary tissue [29] and rat trigeminal mesencephalic nucleus neurons [30], suggesting that $A S I C 1 b$ is present in certain brain regions. A variety of techniques have been used to demonstrate that both $A S I C 2 a$ and $A S I C 2 b$ show diffuse expression throughout the brain in a number of species, as well as the spinal cord and DRG [26, 31-33]. By contrast, initial work using northern blot and in situ hybridization suggested that in rats ASIC3 was only present in DRG and was absent from the brain [34]. However, later work using a combination of western blot and RT-PCR showed that it was present in hippocampus, amygdala, caudate putamen, prefrontal cortex and hypothalamus, as well as and DRG [35]. Initial studies examining the expression of ASIC4 using dot blot, northern blot and RT-PCR showed that it was present in the brain, spinal cord and DRG [36, 37], work that has been supported by more recent investigation using ASIC4 transgenic marker mice [8]. By contrast with mice and rats, there is very little known about ASIC expression in naked mole-rats, although one study used RT-PCR to demonstrate that transcripts for ASIC1a, ASIC1b, ASIC2a, ASIC2b and ASIC3 are present in DRG, but expression of ASIC4 was not determined [14].

Overall, reports of ASIC expression lack consistency of methodology, both in selection and comparison of tissue and the method used to report quantities. To overcome these shortcomings, we developed a rigorous qPCR protocol using appropriate normalization and a large number of different tissues. RNA was extracted from mouse and naked mole-rat brain (olfactory bulbs, cerebellum, brain stem, cerebral cortex and hippocampus), spinal cord and DRG and cDNA produced. A species-specific geNorm assay was then used to find reliable housekeeping genes, which enabled a reliable and comparable estimate of the relative amounts of $A S I C$ transcripts present.

\section{Methods}

\section{Animals}

All experiments were conducted in accordance with the United Kingdom Animal (Scientific Procedures) Act 1986 under a Project License (70/7705) granted to E. St. J. S. by the Home Office; the University of Cambridge Animal Welfare Ethical Review Body also approved procedures. Young adult animals were used in this study: male and female, 7-week-old C57/bl6 mice and 6-month old male and female naked mole-rats; considering the $\sim 30$ year life span of naked mole-rats, 6-month old animals can be considered young adults like the 7-week old mice. Mice were housed in groups of up to five mice per cage with nesting material and a red plastic shelter; the holding room was temperature controlled $\left(21^{\circ} \mathrm{C}\right)$ and mice were on a standard 12-h light/dark cycle with food and water available ad libitum. Naked mole-rats were bred in house and maintained in a custom-made caging system with conventional mouse/rat cages connected by different lengths of tunnel. Bedding and nesting material were provided along with a running wheel. The room was warmed to $28{ }^{\circ} \mathrm{C}$, with a heat cable to provide extra warmth running under 2-3 cages, and red lighting (08:00-16:00) was used.

\section{RNA extraction and CDNA synthesis}

Animals were decapitated and tissues were rapidly dissected under a sterile hood using sterilized dissection tools and immediately transferred to TRI reagent (Sigma, $1 \mathrm{ml}$ for $100 \mathrm{mg}$ tissue) in $1.5 \mathrm{ml}$ tubes. Samples were stored at $-80{ }^{\circ} \mathrm{C}$ until further use. Once fully defrosted, tissues were homogenized using Eppendorf micropestles and incubated for $5 \mathrm{~min}$ at room temperature (RT). After adding 1:10 volume of 1-bromo-3-chloropropane and shaking for $15 \mathrm{~s}$, samples were incubated another $3 \mathrm{~min}$ at $\mathrm{RT}$ and then centrifuged at $10,000 \mathrm{rpm}$ for $15 \mathrm{~min}$ at $4^{\circ} \mathrm{C}$ to separate the mixture into three phases: a red organic phase containing protein, an interphase containing DNA and a colorless aqueous phase containing RNA. The aqueous phase was transferred to a new $1.5 \mathrm{ml}$ tube and $500 \mu \mathrm{l}$ $100 \%$ ethanol were added. The mixture was then applied to a Zymo RNA Clean \& Concentrator column and an incolumn DNaseI digest and clean-up of RNA were performed according to kit instructions. The RNA was eluted 
in $20 \mu \mathrm{l}$ and a $3 \mu \mathrm{l}$ aliquot was taken before storing at $-80{ }^{\circ} \mathrm{C}$. To test the quality of the extracted RNA, $1.5 \mu \mathrm{l}$ of the aliquot were used to determine the concentration, while the remaining $1.5 \mu \mathrm{l}$ were used for gel electrophoresis. To test the integrity and quality of RNA, $500 \mathrm{ng}$ samples were run on a $1 \%$ agarose gels with TAE buffer, RNA quality assessed by identification of the $28 \mathrm{~S}, 18 \mathrm{~S}$ and $5 \mathrm{~S}$ ribosomal RNA bands on a $1 \%$ agarose gel; in the case of naked mole-rat RNA, one additional band is expected resulting from the split $28 \mathrm{~S}$ subunit [38]. In addition, samples were measured using RNA spectroscopy (NanoVueTM, VWR). RNA was transcribed into cDNA using random nonamer and $\mathrm{dT}$ oligomer priming with the Precision nanoScript 2 kit (Primerdesign, for qPCR) according to the manufacturer's directions.

\section{Primer design}

Naked mole-rat ASIC and mouse ASIC1b primers were designed with the help of the PrimerQuest online tool (www.idtdna.com/PrimerQuest/) with the specification that the melting temperature be $57{ }^{\circ} \mathrm{C}$, the GC content $45-55 \%$ and the product length $100-200 \mathrm{bp}$. Housekeeping primers and all other mouse ASIC primers were designed and tested by Primerdesign adhering to the same specifications Tables 1, 2 and 3). Primers were first tested by performing a standard RT-PCR using DreamTaq polymerase, each $20 \mu \mathrm{l}$ reaction containing $1 \mathrm{X}$ DreamTaq reaction buffer, $200 \mu \mathrm{M}$ of dATP, dTTP, dGTP and dCTP, $0.5 \mu \mathrm{M}$ of each primer, $10 \mathrm{ng}$ cDNA and $0.02 \mathrm{U} / \mu \mathrm{l}$ DreamTaq DNA Polymerase and the PCR was run with the conditions $95^{\circ} \mathrm{C} 5 \mathrm{~min}, 40 \mathrm{X}$ $\left(95{ }^{\circ} \mathrm{C} 30 \mathrm{~s}, 60{ }^{\circ} \mathrm{C} 30 \mathrm{~s}, 72{ }^{\circ} \mathrm{C} 15 \mathrm{~s}\right), 72{ }^{\circ} \mathrm{C} 5 \mathrm{~min}$, cool to $12{ }^{\circ} \mathrm{C}$. The resulting bands were visualized on a $1 \%$ agarose gel to test primer specificity. Primers with only one product were deemed specific and were then tested in a qPCR reaction. For this validation, a standard curve experiment with RNA amounts of $25 \mathrm{ng}, 12.5 \mathrm{ng}, 5 \mathrm{ng}, 2.5$ $\mathrm{ng}, 1.25 \mathrm{ng}$ and $0.5 \mathrm{ng}$ was performed and a linear regression calculated for each primer pair. From the slope of this regression, the amplification factor (AF) and efficiency (E) could be calculated using the formula: $\mathrm{AF}=10^{-(1 / \text { slope })}$ and $\mathrm{E}=\mathrm{AF}-1 \times 100$. Only primers with an efficiency of $70-130 \%$ were used in subsequent experiments.

\section{qPCR}

For all qPCR experiments, cDNA equivalent to $25 \mathrm{ng}$ of RNA were used per reaction, to which $15 \mu \mathrm{l}$ of reaction mix $(10 \mu$ master mix precisionPLUS from Primerdesign, $4 \mu \mathrm{H}_{2} \mathrm{O}, 1 \mu \mathrm{l}$ gene specific primers from Primerdesign or Sigma-Aldrich) were added. For each gene of interest, a water sample was loaded as negative control, containing $5 \mu \mathrm{H}_{2} \mathrm{O}$ and $15 \mu \mathrm{l}$ reaction mix. For each sample, two technical replicates were included.
Table 1 geNorm primer binding locations

\begin{tabular}{|c|c|c|c|c|}
\hline Species & Gene & Accession no. & $\begin{array}{l}\text { Anchor } \\
\text { nucleotide }\end{array}$ & $\begin{array}{l}\text { Sequence } \\
\text { length (bp) }\end{array}$ \\
\hline \multirow[t]{12}{*}{ Mouse } & 185 & NR_003278.3 & 134 & 99 \\
\hline & ACTB & NM_007393.3 & 597 & 94 \\
\hline & ATP5b & NM_016774.3 & 1115 & 142 \\
\hline & $B 2 M$ & NM_009735.3 & 202 & 159 \\
\hline & CANX & NM_007597.3 & 2827 & 127 \\
\hline & $\mathrm{CYCl}$ & NM_025567.2 & 514 & 203 \\
\hline & EIF4A2 & NM_013506.2 & 876 & 215 \\
\hline & GAPDH & NM_008084.2 & 793 & 180 \\
\hline & RPL13A & NM_009438.5 & 691 & 180 \\
\hline & SDHA & NM_023281.1 & 2018 & 181 \\
\hline & $U B C$ & NM_019639.4 & 2225 & 178 \\
\hline & YWHAZ & NM_011740.3 & 1045 & 195 \\
\hline \multirow[t]{11}{*}{ Naked mole-rat } & ACTB & XM_004840381 & 1231 & 143 \\
\hline & $B 2 M$ & XM_013078376 & 128 & 116 \\
\hline & CANX & XM_013077439 & 1068 & 139 \\
\hline & EIF4A2 & XM_004834818 & 404 & 145 \\
\hline & GAPDH & XM_004869398 & 455 & 118 \\
\hline & GPI & NM_001310272 & 1746 & 101 \\
\hline & $\mathrm{MDH} 1$ & XM_004849985 & 548 & 112 \\
\hline & RPL13A & XM_004866919 & 362 & 92 \\
\hline & SDHA & XM_004845101 & 784 & 179 \\
\hline & TOP1 & XM_004874029 & 128 & 116 \\
\hline & YWHAZ & XM_004850171 & 926 & 79 \\
\hline
\end{tabular}

Table of specifications for primer binding according to Primerdesign, showing the sequence length in base pairs, the anchor nucleotide (central to the binding region) and accession number of the gene

\section{geNorm analysis}

geNorm analysis was performed using a commercially available primer kit for mouse and a custom-made primer kit for naked mole-rat, both by Primerdesign. For both analyses, 12 housekeeping gene-specific primer pairs were used and the expression of the genes was determined in two biological samples from each tissue using $\mathrm{qPCR}$ as described above. The geNorm software (part of qbase+, https://www.qbaseplus.com) calculated the pair-wise expression ratio of all genes, the variation of which corresponds to expression stability, resulting in a ranking of the genes based upon a gene stability measure $(\mathrm{M})$. geNorm calculates how many housekeeping genes should be used for normalization by calculating a normalization factor and pairwise variation $(\mathrm{V})$, which asks how the addition of the next best housekeeping genes impacts the stability of the normalization.

\section{$\Delta \mathrm{CT}$ analysis}

For analysis of $A S I C$ expression, the $\triangle \mathrm{CT}$ (threshold cycle) method was used [39]. CT values were normalized by 
Table 2 Mouse qPCR primers

\begin{tabular}{lllll}
\hline Gene & Accession no. & Primer fw & Primer rv & Sequence length (bp) \\
\hline ASIC1a & NM_009597 & gaactgaagaccgaggaggag & gccgctcataggagaagatgt & 112 \\
ASIC1b & NM_001289791 & tcagctaccctgacttgctcta & gagcggttgtagaaacgatgga & 139 \\
ASIC2a & NM_001034013 & cgatggacctcaaggagagc & atacacgaagatgtggcggat & 107 \\
ASIC2b & NM_007384 & cttgctgttgtcctggtcct & ttgttgttgcacacggtgac & 123 \\
ASIC3 & NM_183000 & ttcacctgtcttggctcctc & tgactggggatgggatttctaag & 126 \\
ASIC4 & NM_183022 & caccttgctggagatccttga & gtccgcagtggggtcttg & 150
\end{tabular}

Forward ( $\mathrm{fw}$ ) and reverse ( $\mathrm{rv}$ ) primers in 5'-3' orientation, accession number and length of product in base pairs

subtracting the geometrical mean of the reference $\mathrm{CT}$ values from the sample $\mathrm{CT}$ value $\left(\mathrm{CT}_{\text {sample }}-\mathrm{CT}_{\text {ref }}=\right.$ $\Delta C T$ ). The efficiency (E) was then used to determine the normalized relative abundance of transcripts by calculating $\mathrm{E}^{-\Delta \mathrm{CT}}$ and this value was plotted on a $\log _{10}$ axis to visualize the exponential nature of the fold change between samples/transcripts. Statistical analysis within tissues and between species has not been conducted because although primer efficiencies are comparable throughout, conditions between reactions and tissues are too different to allow for such a comparison.

\section{Results}

\section{RNA integrity}

As an estimate for RNA purity and integrity, spectroscopy values and agarose gel electrophoresis were used. Following spectroscopy analysis, and only samples with a A260/ A280 ratio $>1.8$ were used, which is an indication that they contained little to no contamination [40]. Ribosomal RNA is the most abundant and as expected was visible in the samples after DNaseI treatment and clean-up. Electrophoresis of mouse samples showed $28 \mathrm{~S}$ and $18 \mathrm{~S}$ ribosomal subunits (Fig. 1a), whereas naked mole-rat samples showed three bands as expected due to cleavage of the $28 \mathrm{~S}$ subunit (Fig. 1b) [38].

\section{geNorm}

To find a combination of housekeeping genes that was most stably expressed between all sampled tissues, a geNorm analysis was performed. This analysis resulted in two plots, the first of which shows a ranking of all genes tested in order of expression stability (M) between tissues, with less variation (i.e. higher stability) resulting in a lower $M$ value (Fig. 2a and c). The second plot shows pairwise $V$ numbers, a value that expresses how reliable normalization is by using two housekeeping genes rather than three $(\mathrm{V} 2 / 3)$ and how this reliability changes by adding more genes one by one (V3/4, V4/5, and so on, Fig. $2 \mathrm{~b}$ and $\mathrm{d})$. In both species, a reliable normalization could be achieved using the two most stably expressed housekeeping genes, which were RPL13A (ribosomal protein L13A) and CANX (calnexin) in mouse (Fig. 2a) and $\beta$-ACTIN and EIF4A (eukaryotic initiation factor 4a) in naked mole-rat (Fig. 2c). In the naked mole-rat tissues, only three genes were stably expressed in all tissues (Fig. 2c), and using more than two housekeeping genes for normalization would have added noise to the results (Fig. 2d) and thus for both mouse and naked mole-rat we used two housekeeping genes for normalization.

\section{qPCR}

Relative expression fold change for different ASIC transcripts was determined using the $\triangle C T$ method (see Methods) in both mouse and naked mole-rat. In the mouse, all ASICs were expressed in all tissues analyzed, but at distinctly different levels (Fig. 3). ASIC3 was expressed in all tissues analyzed at comparable levels apart from in the DRG where ASIC3 levels were approximately 10-fold higher than in all other tissues (Fig. 3a). In the spinal cord and across most brain regions, ASIC3 was expressed at a similar level to $A S I C 1 a$ and $A S I C 2 b$ with $A S I C 1 b$ and $A S I C 2 a$ showing consistently lower expression (Fig. $3 \mathrm{~b}-\mathrm{g}$ ). Thus in the mouse brain $A S I C$ expression can be split into two groups, high expression: ASIC1a, ASIC2b and ASIC3, and low

Table 3 Naked mole-rat qPCR primers

\begin{tabular}{lllll}
\hline Gene & Accession no. & Primer fw & Primer rv & Sequence length (bp) \\
\hline ASIC1a & XM_013078965.1 & atgagataccagacacgcagat & gcagcatgtctcgaatgtcatg & 144 \\
ASIC1b & NM_001279840 & ggtgccagtcatgtctttgtg & catgcgggtagctgaggtaata & 136 \\
ASIC2a & XM_013067767 & gcacgttaccaaggtggatgag & tggtggtgagcctggagaa & 101 \\
ASIC2b & XM_004870614.2 & tcgaaccgcctgctgtact & gggttgttattgcacacggtga & 107 \\
ASIC3 & 00000022115 & atccgagtgcagatccacag & gttcctcaaagtcggagtccat & 172 \\
ASIC4 & XM_004864511.1 & ccagcaacttctctgtggtctat & actcctcctgctggatgtcta & 163 \\
\hline
\end{tabular}

Forward (fw) and reverse (rv) primers in $5^{\prime}-3^{\prime}$ orientation, accession number and length of product in base pairs 

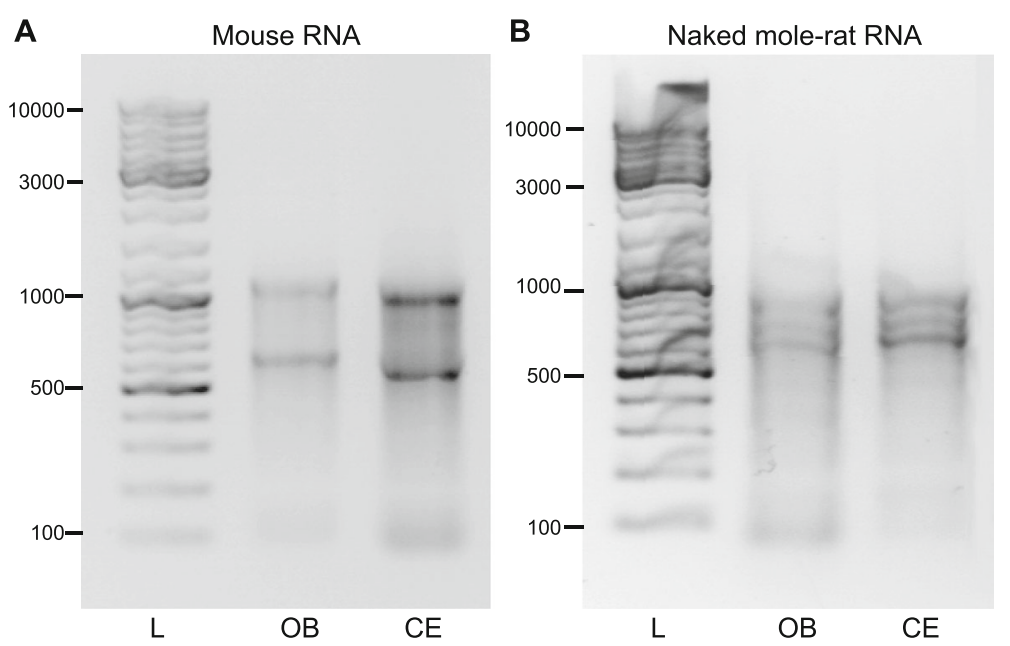

Fig. 1 RNA samples on a 1\% agarose gel after DNasel digest and clean up. a Mouse RNA from olfactory bulb (OB) and cerebellum (CE), the DNA ladder (L) marks DNA sizes from 100bp to 10,000bp. b Naked mole-rat RNA from OB and CE

expression: $A S I C 1 b$ and $A S I C 2 a$. With the exception of DRG and the hippocampus, where ASIC4 showed the lowest level of expression, ASIC4 was frequently the most highly expressed ASIC (Fig. 3a - g).

In naked mole-rat tissues, a similar pattern was observed for ASIC DRG expression as in mouse, ASIC3 was the most highly expressed transcript and ASIC4 the lowliest (Fig. 3a'). Similarly, as in mouse tissues, in naked molerat non-DRG tissue, expression could be grouped into a high expression group (ASIC1a, ASIC2b and ASIC 3 ) and a low expression group (ASIC1b and ASIC2a) (Fig. 3b' - g'). However, by contrast with the mouse expression profile, ASIC4 showed the lowest level of expression in all tissues (Fig. 3a' - g').

\section{Discussion}

\section{geNorm analysis identified optimal combination of} housekeeping genes

A geNorm analysis was performed to identify the most stably expressed housekeeping genes. The gene stability value $(\mathrm{M})$ is based on the assumption that the expression ratio between two control genes should be the same in all samples, therefore increasing variation in ratio means decreasing expression stability. Average M-values are usually in the range of $0.5-1$ when using heterogeneous tissue samples, and values $>1$ indicate low stability $[41,42]$. The geNorm algorithm ranked the twelve control genes according to their pairwise expression stability in five brain regions, spinal cord and DRG neurons. Studies have found that using two older programs, BestKeeper or NormFinder, which are based on the same paradigm, but use slightly different algorithms, results in significantly similar gene rankings, emphasizing the validity of the results using geNorm [43].
Analysis of human and mouse qPCR studies in the literature found that the most frequently used housekeeping genes were GAPDH, $18 S$ and $\beta$-ACTIN, however, evidence suggests that they might not be appropriate for all tissues and conditions, especially during development, and therefore it is important to determine the best housekeeping gene combination at the start of a qPCR study [44-47]. Moreover, no study has investigated optimal housekeeping genes for experiments using naked mole-rat tissue. As per geNorm guidelines, the optimal number of reference genes is reached when the $\mathrm{V}$ (pairwise variation) value falls below 0.15 [42]. In both mouse and naked mole-rat, using two reference genes for normalization fulfilled this criterion.

The stability analysis showed many similarities, but also some incongruence between the two species. It should firstly be noted that although rodents, mice and naked mole-rats belong to two different Rodentia suborders, Myomorpha and Hystricomorpha respectively and thus differences in gene stability are perhaps to be expected. Moreover, age is also a complicating factor because although adult mice (6-7-month old) were used, it is not entirely clear how the developmental stage relates to 6-month old naked mole-rat, which can live for $\sim 30$ years, although at 4-months of age the naked mole-rat brain is already $90 \%$ of the adult size [48]. The development of the eusocial naked mole-rat is somewhat different because animals do not sexually mature unless they are part of the breeding pair or are removed from the influence of the breeding female [49]. However, the animals used in this study can be considered adults at this age, because they can mature and reproduce if separated from the colony.

Although a previous study has identified $\beta$-2-microglobulin $(B 2 M)$ as being the most stably expressed transcript in different mouse brain regions when comparing neonatal and adult tissue [40], we found $B 2 M$ to be the most variable gene 

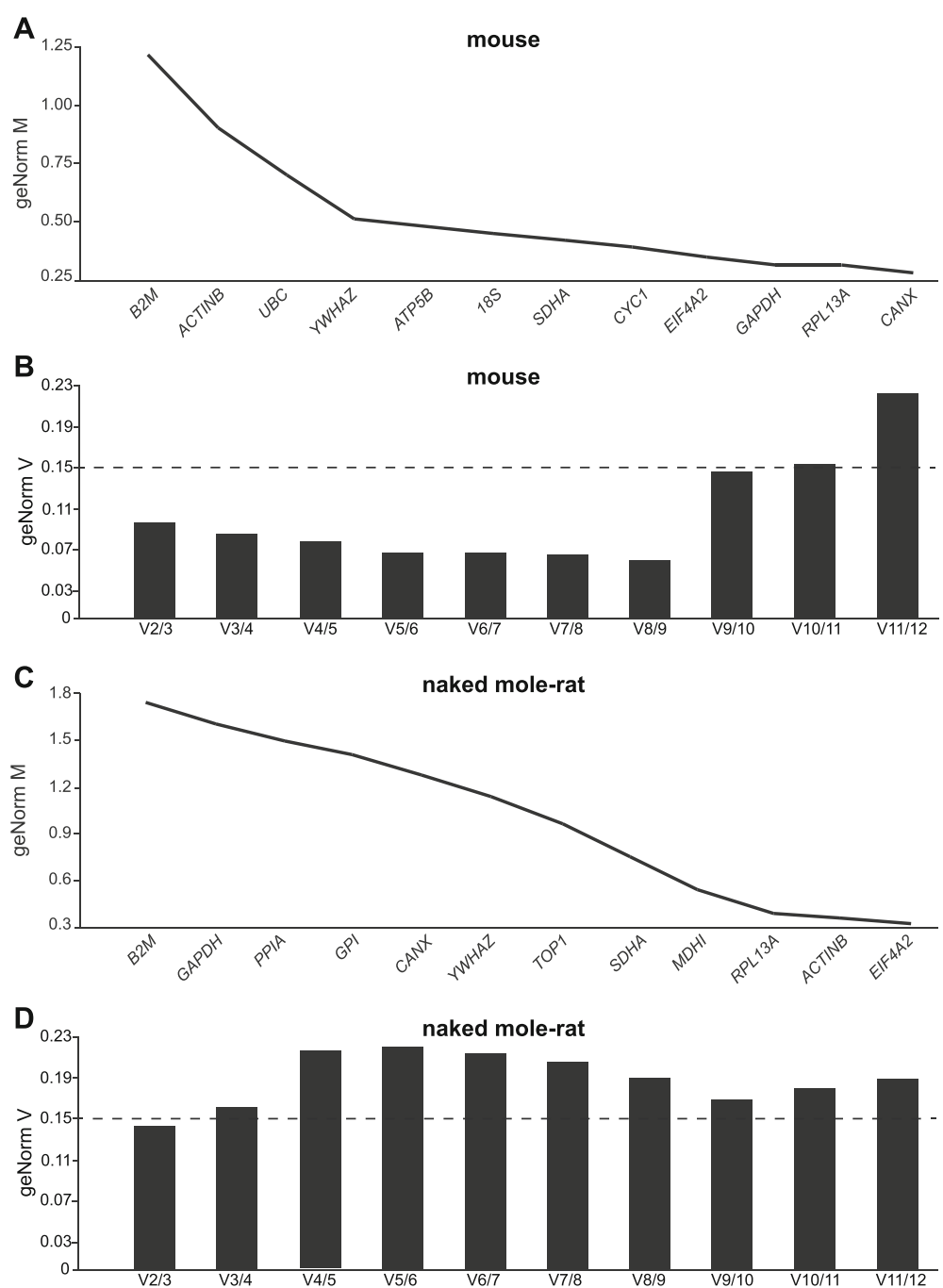

Fig. 2 Ranking of housekeeping genes based on their expression stability between tissue samples (2 biological replicates per tissue), from low stability (left) to high stability (right). a Mouse geNorm experiment showing CANX and RPL13A to be the most stably expressed transcripts; M represents variation between samples based on pairwise expression ratio, a lower value means lower variability. $\mathbf{b}$ Mouse data showing the optimal number of reference genes for an experiment using samples from all tissues analyzed; $V$ indicates if addition of more housekeeping genes, starting with the two most stable genes from plot 1, increases or decreases variation (a value below 0.15 is recommended). c Naked mole-rat geNorm experiment (2 biological replicates per tissue), EIF4A2 and $\beta$-ACTIN were the most stably expressed transcripts. $\mathbf{d}$ Naked mole-rat data showing the optimal number of reference genes for an experiment using samples from all tissues analyzed

in both species (Fig. 2a and c), indicating that although perhaps useful for comparing developmental stages, $B 2 M$ should not be used for normalization of qPCR experiments using adult mouse and naked mole-rat brain tissues. Overall, RPL13A and EIF4A2 were within the four most stable genes in both species and therefore could be used for future interspecies comparisons.

Bruckert et al. tested the expression of nine commonly used reference genes in four mouse brain regions, including some of the same structures examined in our study (cortex, cerebellum and hippocampus) at different stages of development and found that at least two reference genes were needed for good normalization [50]. Interestingly, in three of the tested tissues (cortex, hippocampus, striatum), RPL13A was the most stable reference gene [50], and RPL13A was found to be a robust housekeeping gene in mouse samples in this study, too (Fig. 2a).

Although widely used as a reference housekeeping genes, Kouadjo et al. found that glyceraldehyde-3-phosphate dehydrogenase (GAPDH) and $\beta$-ACTIN can have significantly different expression between different tissues and may not be reliable in studies using very diverse tissue types [51]. Here, we find that GAPDH is stably expressed in mouse tissues, but not in naked mole-rat tissues, whereas the trend 







\section{(See figure on previous page.)}

Fig. 3 Expression ASIC transcripts in mouse and naked mole-rat tissues. Plots show mean and standard error of the mean (SEM) of $\log _{10}$ of ASIC transcripts normalized to the CANX and RPL13A for mouse tissues and ACTINB and EIF4A2 for naked mole-rat tissues. a and $\mathbf{a}^{\prime}$ DRG (mouse, $\mathrm{n}=3$, naked mole-rat, $n=4$ ), $\mathbf{b}$ and $\mathbf{b}^{\prime}$ spinal cord (mouse, $n=7$, naked mole-rat, $n=3-4$ ), $\mathbf{c}$ and $\mathbf{c}^{\prime}$ cortex (mouse, $n=4-5$, naked mole-rat, $n=4$ ), $\mathbf{d}$ and $\mathbf{d}^{\prime}$ hippocampus

(mouse, $n=3$, naked mole-rat, $n=3-4$ ), e and $\mathbf{e}^{\prime}$ olfactory bulb (mouse, $n=6-8$, naked mole-rat, $n=3-4$ ), $\mathbf{f}$ and $\mathbf{f}$ cerebellum (mouse, $n=7$, naked mole-rat, $n=4$ ), and $\mathbf{g}$ and $\mathbf{g}^{\prime}$ brain stem (mouse, $n=6$, naked mole-rat, $n=3-4$ ). $n=$ number of biological replicates

is reversed for $\beta$-ACTIN such that it is the second most stably expressed in gene in naked mole-rat tissue, but the second most unstable in mouse tissue (Fig. 2a and c). These results clearly indicate the variability of these two genes between species and tissues and demonstrate the importance of conducting gene stability analysis before choosing appropriate housekeeping genes. Interestingly, $\beta$-ACTIN is under positive selection in the naked mole mole-rat [52] and the resulting variation in this highly conserved gene might make the protein more resistant to oxidation. It can thus be speculated that expression is also affected, which introduces the possibility that $\beta$-ACTIN expression is more stable in the naked mole-rat brain than in the brains of other rodents and this increased stability may account for $\beta$-ACTIN being the second most stably expressed in gene in naked mole-rat tissue.

CANX has been previously described as a stable reference gene between germ and somatic cells of both sexes in mice when using fetal cells [53] and a further geNorm study using eight genes on the liver tissue from the nonmodel mouse species Apodemos flavicollis (yellownecked mouse) found CANX to be consistently within the three most stable genes [43]. In the current geNorm study, we demonstrate that $C A N X$ was the most stably expressed gene between mouse tissues, but this was not the case for naked mole-rat tissues (Fig. 2a and c). By contrast, Axtner et al. found ubiquitin $\mathrm{C}(U B C)$, ribosomal protein L13A (RPL13A) and $\beta$-ACTIN to be the least stable genes they examined [43], whereas RPL13A and $\beta$-ACTIN were two of the three most stably expressed genes in naked mole-rat samples (Fig. 2c) and RPL13A the second most stable mouse housekeeping gene, however, in agreement with Axtner et al., $\beta$-ACTIN and $U B C$ were two of the three least stable genes in mouse tissues (Fig 2a). Although some studies have warned that $\beta$-ACTIN is not always an appropriate gene to use for normalization because its expression can be affected by treatments and age, a majority of studies analyzed by Chapman et al. found $\beta$-ACTIN to be among the most stable genes in the brain, especially in the hippocampus, cortex, basal ganglia, mesencephalon, but also whole brain samples [46]. It is therefore not unusual that $\beta$-ACTIN was one of the two most stable reference genes in the naked mole-rat brain. The huge variation in stability of housekeeping genes between different studies clearly shows the importance of performing a geNorm (or similar) analysis at the start of each study, using the same primers and samples that will be used for subsequent experiments.

In summary, we determined that RPL13A and CANX were the most stable housekeeping genes in mouse tissues (3rd and 11th in naked mole-rat) and that $A C T I N B$ and EIF4A2 were the most stable housekeeping genes in naked mole-rat tissues (11th and 4th in mouse).

\section{qPCR expression confirmed ASIC expression in the nervous system}

Previous studies of ASIC expression relied heavily on either RNA-oligonucleotide-based (such as in situ hybridization and Northern Blot) or on antibody-based (immunohistochemistry, Western Blot) techniques. However, many studies either failed to differentiate between splice isoforms or did not determine the expression of every ASIC subunit (Table 4).

We have previously shown that $A S I C 3$ is most abundant in mouse DRG neurons, $A S I C 1 b, A S I C 2 a$ and $A S I C 2 b$ having similar levels and ASIC1a being least abundant (ASIC4 was not investigated) [14]. Here we find that ASIC3 is most abundant, but that $A S I C 1 b$ rather than $A S I C 1 a$ is least abundant, although both $A S I C 2 a$ and $A S I C 2 b$ have higher expression in accordance with our earlier study (Fig. 3a). However, it should be noted that our previous study only used one housekeeping gene for normalization (HPRT1, hypoxanthine phosphoribosyltransferase 1). A comparison of ASICla and $A S I C 1 b$ expression in human DRG also found that $A S I C 1 b$ was higher in DRG neurons than ASIC1a, but again a single reference gene was used (GAPDH) [54]. We also find that ASIC4 is the least abundant ASIC in DRG, which is in accordance with previous data showing that ASIC4 is sparsely expressed in DRG $[8,36,37]$.

In accordance with previous accounts, ASIC1a, ASIC1b, $A S I C 2 a$ and $A S I C 2 b$ were expressed abundantly in the brain and spinal cord of mice $[26,27,55]$. ASIC1b expression was consistently lower than ASIC1a, which might explain why it was previously thought that this subunit was confined to DRG [28]. In a qPCR study comparing ASIC expression in the mouse spinal cord, $A S I C 2 a$ was most highly expressed followed by ASIC1a, while the expression of ASIC3 was rather low [33], whereas we find that $A S I C 2 a$ is the lowest of the these transcripts (Fig. 3b). However, it is difficult to compare our results with those of Baron et al. because no error bars are provided in their data and their study only used a single reference gene, $18 \mathrm{~S}$, which for reasons discussed previously may not be the most appropriate reference gene to use. 
Table 4 ASIC expression in the nervous system

\begin{tabular}{|c|c|c|c|c|c|c|c|c|}
\hline & DRG & SP & $\mathrm{CO}$ & $\mathrm{HC}$ & $\mathrm{OL}$ & $\mathrm{CE}$ & BS & Whole brain \\
\hline$\overline{A S I C 1}$ & NB (r[28]) & NB (r[28]) & $\begin{array}{l}\text { ISH (m[27], } \\
\text { r[58]) } \\
\text { NB (r[28]) } \\
\text { IHC (m[7]) }\end{array}$ & $\begin{array}{l}\text { ISH (m[27, 25], } \\
\text { r[58]) IHC (m[7]) } \\
\text { NB (r[28], h[25]) }\end{array}$ & $\begin{array}{l}\text { ISH (m[27, 25], } \\
\text { r[58]), } \\
\operatorname{IHC~(m[7])~}\end{array}$ & $\begin{array}{l}\text { ISH (m[27, 25], } \\
\text { IHC (m[7]), } \\
\text { NB (r[28]) }\end{array}$ & NB (r[28]) & $\begin{array}{l}\text { NB }(r[28]), \\
\text { ISH (m[25]) }\end{array}$ \\
\hline ASIC1a & $\begin{array}{l}\text { qPCR } \\
\text { (h[31, 54], } \\
\mathrm{m}[14,54], \\
\mathrm{nmr}[14])\end{array}$ & $\begin{array}{l}\text { qPCR (h[31], } \\
\mathrm{m}[33]\end{array}$ & & & & & & $\begin{array}{l}\text { qPCR } \\
(\mathrm{h}[31,54], \mathrm{m}[54])\end{array}$ \\
\hline ASIC1b & $\begin{array}{l}\text { qPCR } \\
(m[14,54], \\
n m r[14])\end{array}$ & & & & & & & qPCR (m[54]) \\
\hline $\mathrm{ASIC2}$ & NB (r[36]) & NB (r[36]) & ISH (r[26, 36, 58]) & $\begin{array}{l}\text { ISH (m[25], } \\
\mathrm{r}[26,36,58]) \\
\text { NB }(\mathrm{h}[25]) \\
\text { EP (m[11]) }\end{array}$ & $\begin{array}{l}\text { ISH }(\mathrm{m}[25] \\
\mathrm{r}[26,36,58])\end{array}$ & $\begin{array}{l}\text { ISH (m[25], } \\
r[26,36] \\
\text { NB }(r[36])\end{array}$ & NB (r[36]) & $\begin{array}{l}\text { NB (r[36]) } \\
\text { ISH (m[25]) }\end{array}$ \\
\hline ASIC2a & $\begin{array}{l}\text { qPCR (m[14], } \\
n m r[14])\end{array}$ & qPCR (m[33]) & & & & & & qPCR (h[31]) \\
\hline$A S I C 2 b$ & $\begin{array}{l}\mathrm{qPCR}(\mathrm{m}[14] \\
\mathrm{nmr}[14])\end{array}$ & qPCR (m[33]) & & & & & & qPCR (h[31]) \\
\hline ASIC3 & $\begin{array}{l}\text { RT (r[35]), } \\
\text { NB (r[36]), } \\
\text { qPCR (h[31], } \\
\text { r[31], m[14], } \\
\text { nmr[14]) } \\
\text { WB (r[35]) }\end{array}$ & $\begin{array}{l}\text { qPCR (h[31], } \\
m[33])\end{array}$ & $\begin{array}{l}\text { RT (r[35]), } \\
\text { WB (r[35]) }\end{array}$ & $\begin{array}{l}\text { RT (r[35]), } \\
\text { WB (r[35]) }\end{array}$ & & $\mathrm{ISH}(\mathrm{r}[58])$ & & qPCR (h[31]), \\
\hline $\mathrm{ASIC} 4$ & & $\begin{array}{l}\text { NB (r[36]) } \\
\text { ISH (r[36]) }\end{array}$ & $\begin{array}{l}\text { ISH (r[36]) } \\
\text { RT (r[37]) }\end{array}$ & ISH (r[36]) & & $\begin{array}{l}\text { NB (r[36]) } \\
\text { RT (r[37]) }\end{array}$ & $\begin{array}{l}\text { NB (r[36]) } \\
\text { RT (r[37]) }\end{array}$ & $N B(r[36,37])$ \\
\hline
\end{tabular}

Different techniques have been previously used to determine ASIC expression in the nervous system tissues used in this study. Abbreviations used in Table 4 are as follows $B S$ brain stem, $C E$ cerebellum, CO cortex, DRG dorsal root ganglia, EP electrophysiology, $H C$ hippocampus, IHC immunohistochemistry, ISH in situ hybridisation, NB Northern blot, $O L$ olfactory bulb, $q P C R$ quantitative real-time PCR, $R T$ reverse-transcriptase PCR, WB western blot. Species: $r$ rat, $h$ human, $m$ mouse, $n m r$ naked mole rat. Studies listed for ASIC1 and ASIC2 did not differentiate between splice isoforms

With regards to levels of $A S I C$ transcripts in the brain, ASICla is consistently highly expressed (Fig. 3c - g), which is to be expected considering the importance of ASIC1a in neuronal physiology and animal behavior $[5-7,11,12,56]$. ASIC1b expression is along with that of ASIC2 $a$ commonly the lowliest expressed across different brain regions, whereas $A S I C 2 b$ shows similar levels to ASIC1a. Previous reports have reached little consensus about ASIC3 expression in the brain, but here we demonstrate that ASIC3 is strongly expressed in all tissues investigated, and a RT-PCR study by Meng et al also showed ASIC3 transcripts present in different parts of the brain, in addition to DRGs [35]. Lastly, as others have shown with a variety of techniques $[8,36,37], A S I C 4$ was highly expressed in all brain regions analyzed as well as the spinal cord, where it was more abundant than other ASICs.

To date, nothing is known about ASIC expression in the naked mole-rat brain. However, considering the variable roles of ASICs in neuronal physiology and behavior, it is important for ASIC expression to be understood in the naked mole-rat as differential ASIC function and/or expression could alter neuronal acid sensitivity. This is of particular importance in a species that is known to be adapted to its hypercapnic, subterranean environment, such that a variation in the amino acid sequence of NaV1.7 confers behavioral acid-insensitivity in naked mole-rats $[14,19]$. We have previously shown using qPCR that as in mouse ASIC3 is most abundantly expressed in DRG, followed by ASIC1a and $A S I C 1 b$, with $A S I C 2 a$ and $A S I C 2 b$ being more lowly expressed [14] and in this study we observed an almost identical trend, but with $A S I C 2 a$ actually showing slightly lower expression that $A S I C 2 b$ (Fig. 3a'); we also show for the first time that, as in mouse, ASIC4 is the least abundant ASIC transcript.

When comparing the central nervous system between mouse and naked mole-rat, trends were generally similar, i.e. ASIC1a was the frequently the most highly expressed and $A S I C 1 b$ and $A S I C 2 a$ showing much lower abundance levels (Fig. 3b' - g'). However, the greatest difference observed is that whereas ASIC4 is highly abundant in all mouse central nervous system tissues tested here apart from the hippocampus, in naked mole-rat central nervous system tissues ASIC4 is consistently the least abundant (Fig. 3b' - g'). It has been suggested that ASIC4 plays a modulatory role and can interact with polyubiquitin to downregulate other ASICs [57] and recent evidence suggests that it can counteract the activity of ASICla in the brain to modulate fear and anxiety behavior [8]. Thus, it 




Fig. 4 Summary of ASIC expression determined by QPCR in the olfactory bulb, cerebellum, brain stem, cortex, hippocampus, spinal cord and DRG neurons. Shades of grey indicate the level of ASIC expression in each tissues for mouse and naked mole-rat. For each species, the sample with the highest expression (in both cases ASIC3 in the DRG) was used as a reference (100\% expression) and the relative expression in other tissues was calculated from this for each species independently

can be speculated that one of these functions, or another as of yet unknown function of the subunit, has been lost or made redundant in naked mole-rat, although the physiological properties of naked mole-rat ASIC4 are currently unknown. A summary of ASIC expression in mouse and naked mole-rat tissue is illustrated in Fig. 4 and can be compared to that published by others using a variety of techniques in Table 4.

In conclusion we have conducted a geNorm analysis of mouse and naked mole-rat nervous system tissues to identify appropriate housekeeping genes for normalization. We have then used a robust and uniform strategy to determine ASIC transcript expression in both the mouse and the naked mole-rat. Our results will benefit those who aim to perform qPCR studies in the naked molerat, which is becoming an ever more widely used laboratory species, as well as providing further insight into the role of ASICs.

\section{Abbreviations}

18S: 18s ribosomal subunit; ASIC: Acid-sensing ion channel; ATP5b: ATP synthase, $\mathrm{H}^{+}$transporting, mitochondrial F1 complex, beta polypeptide; B2M: Beta-2-microglobulin; BMR: Blind mole-rat (Spalax galili); bp: Base pairs; BS: Brain stem; cDNA: Complementary DNA; CE: Cerebellum; CNX: Connexin; CO: Cortex; CT: Cycle threshold; CYC1: Cytochrome C1; dATP: Deoxyadenosine triphosphate; dCTP: Deoxycytidine triphosphate; dGTP: Deoxy guanosine triphosphate; DNA: Deoxyribonucleic acid; DRG: Dorsal root ganglia; dTTP: Deoxy thymidine triphosphate; E: Efficiency; EIF4A2: Eukaryotic initiation factor 4A2; fw: Forward; g: Gram(s); GAPDH: Glyceraldehyde 3-phosphate dehydrogenase; GPI: Glucose-6-phosphate isomerase; HC: Hippocampus; L: Ladder; M: geNorm gene-stability measure; mRNA: Messenger RNA; n: Number of biological replicates; NaV: Voltage-gated sodium channel; OB: Olfactory bulb; PCR: Polymerase chain reaction; RPL13A: Ribosomal protein L13a; rRNA: Ribosomal RNA; RT: Room temperature; RT-PCR: Reversetranscriptase PCR; rv: Reverse; SDHA: Succinate dehydrogenase complex, subunit A; SEM: Standard error of the mean; SP: Spinal cord; TM: Melting 
temperature; TRPV1: Transient receptor potential cation channel subfamily $\mathrm{V}$ member 1; UBC: Ubiquitin $C_{;}$V: geNorm pairwise variation measure; YWHAZ: Tyrosine 3-monooxygenase/tryptophan 5-monooxygenase activation protein zeta

\section{Acknowledgements}

We are grateful to the support of animal technicians in particular HR Forest and AJ Robinson. L-N S was awarded Primerdesign's Gold Studentship and thanks A. Davis for technical support.

\section{Funding}

L-N S was a student in the Biotechnology and Biological Sciences Research Council Doctoral Training Program [Grant BB/J014540/1] and a recipient of the David James Pharmacology Award. E St. J S was supported by a Royal Society Research Grant (RG130110).

\section{Availability of data and materials}

The datasets generated and/or analyzed during the current study are available through the Cambridge Research Data Management website, http://dx.doi.org/10.17863/CAM.6000

\section{Authors' contributions}

L-N S and E St. J S conceived and designed the study. L-N S performed the experiments and analyzed the data. L-N S and E St. J S wrote the manuscript. Both authors read and approved the final manuscript.

\section{Competing interests}

The authors declare that they have no competing interests.

\section{Consent for publication}

N/A

\section{Ethics approval and consent to participate}

All experiments were conducted in accordance with the United Kingdom Animal (Scientific Procedures) Act 1986 under a Project License (70/7705) granted to E St. J S by the Home Office; the University of Cambridge Animal Welfare Ethical Review Body also approved procedures.

\section{Author details}

'Department of Pharmacology, University of Cambridge, Tennis Court Road, Cambridge CB2 1PD, UK. ${ }^{2}$ Department of Cell \& Developmental Biology, University College London, Gower Street, London WC1E 6BT, UK.

\section{Received: 24 October 2016 Accepted: 2 December 2016}

Published online: 13 December 2016

\section{References}

1. Lin S-H, Sun W-H, Chen C-C. Genetic exploration of the role of acid-sensing ion channels. Neuropharmacol. 2015;94:99-118.

2. Baron A, Lingueglia E. Pharmacology of acid-sensing ion channels Physiological and therapeutical perspectives. Neuropharmacol. 2015;94:19-35.

3. Gründer S, Pusch M. Biophysical properties of acid-sensing ion channels (ASICS). Neuropharmacol. 2015;94:9-18.

4. Hesselager M, Timmermann DB, Ahring PK. pH Dependency and desensitization kinetics of heterologously expressed combinations of acid-sensing ion channel subunits. J Biol Chem. 2004;279:11006-15.

5. Du J, Reznikov LR, Price MP, Zha X-M, Lu Y, Moninger TO, et al. Protons are a neurotransmitter that regulates synaptic plasticity in the lateral amygdala. Proc Natl Acad Sci U S A. 2014:111:8961-6.

6. Kreple CJ, Lu Y, Taugher RJ, Schwager-Gutman AL, Du J, Stump M, et al. Acid-sensing ion channels contribute to synaptic transmission and inhibit cocaine-evoked plasticity. Nat Neurosci. 2014;17:1083-91.

7. Wemmie JA, Askwith CC, Lamani E, Cassell MD, Freeman JH, Welsh MJ. Acid-sensing ion channel 1 is localized in brain regions with high synaptic density and contributes to fear conditioning. J Neurosci. 2003;23:5496-502.

8. Lin S-H, Chien Y-C, Chiang W-W, Liu Y-Z, Lien C-C, Chen C-C. Genetic mapping of ASIC4 and contrasting phenotype to ASIC1a in modulating innate fear and anxiety. Eur J Neurosci. 2015:41:1553-68.

9. Wu W-L, Lin Y-W, Min M-Y, Chen C-C. Mice lacking Asic3 show reduced anxiety-like behavior on the elevated plus maze and reduced aggression. Genes Brain Behav. 2010;9:603-14.
10. Huda R, Pollema-Mays SL, Chang Z, Alheid GF, McCrimmon DR, Martina M Acid-sensing ion channels contribute to chemosensitivity of breathing-related neurons of the nucleus of the solitary tract. J Physiol. 2012;590:4761-75.

11. Wemmie JA, Chen J, Askwith CC, Hruska-Hageman AM, Price MP, Nolan BC, et al. The acid-activated ion channel ASIC contributes to synaptic plasticity, learning, and memory. Neuron. 2002;34:463-77.

12. Xiong ZG, Zhu XM, Chu XP, Minami M, Hey J, Wei WL, et al. Neuroprotection in ischemia: blocking calcium-permeable acid-sensing ion channels. Cell. 2004;118:687-98.

13. da Silva SI, Husson Z, Bartlett JD, Smith ESJ. Characterization of cutaneous and articular sensory neurons. Mol Pain. 2016;12.

14. Smith ESJ, Omerbašic D, Lechner SG, Anirudhan G, Lapatsina L, Lewin GR. The Molecular Basis of Acid Insensitivity in the African Naked Mole-Rat. Science. 2011;334:1557-60

15. Deval E, Noel J, Lay N, Alloui A, Diochot S, Friend V, et al. ASIC3, a sensor of acidic and primary inflammatory pain. EMBO J. 2008:27:3047-55.

16. Benson CJ, Xie J, Wemmie JA, Price MP, Henss JM, Welsh MJ, et al. Heteromultimers of DEG/ENaC subunits form H+-gated channels in mouse sensory neurons. Proc Natl Acad Sci U S A. 2002;99:2338-43.

17. Omerbašic D, Schuhmacher L-N, Bernal Sierra Y-A, Smith ESJ, Lewin GR ASICS and mammalian mechanoreceptor function. Neuropharmacol. 2015;94:80-6.

18. Deval E, Lingueglia E. Acid-Sensing lon Channels and nociception in the peripheral and central nervous systems. Neuropharmacol. 2015;94:49-57.

19. Park TJ, Lu Y, Juttner R, Smith ES, Hu J, Brand A, et al. Selective inflammatory pain insensitivity in the African naked mole-rat (Heterocephalus glaber). PLoS Biol. 2008;6, e13.

20. Omerbašic D, Smith ESJ, Moroni M, Homfeld J, Eigenbrod O, Bennett NC, et al. Hypofunctional TrkA Accounts for the Absence of Pain Sensitization in the African Naked Mole-Rat. Cell Rep. 2016;17:748-58.

21. Liu Z, Wang W, Zhang T-Z, Li G-H, He K, Huang J-F, et al. Repeated functional convergent effects of NaV1.7 on acid insensitivity in hibernating mammals. Proc Biol Sci. 2014;281:20132950.

22. Schuhmacher L-N, Husson Z, Smith ES. The naked mole-rat as an animal model in biomedical research: current perspectives. Open Acc Anim Phys. 2015;137.

23. Sangameswaran L. A Novel Tetrodotoxin-sensitive, Voltage-gated Sodium Channel Expressed in Rat and Human Dorsal Root Ganglia. J Biol Chem. 1997;272:14805-9.

24. Toledo-Aral JJ, Moss BL, He ZJ, Koszowski AG, Whisenand T, Levinson SR, et al. Identification of PN1, a predominant voltage-dependent sodium channel expressed principally in peripheral neurons. Proc Natl Acad Sci U S A. 1997:94:1527-32.

25. García-Añoveros J, Derfler B, Neville-Golden J, Hyman BT, Corey DP. BNaC1 and $\mathrm{BNaC2}$ constitute a new family of human neuronal sodium channels related to degenerins and epithelial sodium channels. Proc Natl Acad Sci U S A. 1997:94:1459-64.

26. Lingueglia E, de Weille JR, Bassilana F, Heurteaux C, Sakai H, Waldmann R, et al. A modulatory subunit of acid sensing ion channels in brain and dorsal root ganglion cells. J Biol Chem. 1997;272:29778-83.

27. Waldmann R, Champigny G, Bassilana F, Heurteaux C, Lazdunski M. A proton-gated cation channel involved in acid-sensing. Nature. 1997;386:173-7.

28. Chen CC, England S, Akopian AN, Wood JN. A sensory neuron-specific, proton-gated ion channel. Proc Natl Acad Sci U S A. 1998;95:10240-5.

29. Du J, Reznikov LR, Welsh MJ. Expression and Activity of Acid-Sensing lon Channels in the Mouse Anterior Pituitary. Askwith C, editor. PLoS One. 2014:9:e115310

30. Nakamura M, Jang I-S. Characterization of proton-induced currents in rat trigeminal mesencephalic nucleus neurons. Brain Res. 2014;1583:12-22.

31. Delaunay A, Gasull X, Salinas M, Noel J, Friend V, Lingueglia E, et al. Human ASIC3 channel dynamically adapts its activity to sense the extracellular $\mathrm{pH}$ in both acidic and alkaline directions. Proc Natl Acad Sci U S A. 2012;109:13124-9.

32. Price MP, MCllwrath SL, Xie J, Cheng C, Qiao J, Tarr DE, et al. The DRASIC cation channel contributes to the detection of cutaneous touch and acid stimuli in mice. Neuron. 2001:32:1071-83.

33. Baron A, Voilley N, Lazdunski M, Lingueglia E. Acid sensing ion channels in dorsal spinal cord neurons. J Neurosci. 2008;28:1498-508.

34. Waldmann R, Bassilana F, de Weille J, Champigny G, Heurteaux C, Lazdunski M. Molecular cloning of a non-inactivating proton-gated $\mathrm{Na}$ + channel specific for sensory neurons. J Biol Chem. 1997;272:20975-8. 
35. Meng QY, Wang W, Chen XN, Xu TL, Zhou JN. Distribution of acid-sensing ion channel 3 in the rat hypothalamus. Neurosci. 2009;159:1126-34.

36. Akopian AN, Chen CC, Ding Y, Cesare P, Wood JN. A new member of the acid-sensing ion channel family. Neurorep. 2000;11:2217-22.

37. Grunder S, Geissler HS, Bassler EL, Ruppersberg JP. A new member of acidsensing ion channels from pituitary gland. Neurorep. 2000;11:1607-11.

38. Azpurua J, Ke Z, Chen IX, Zhang Q, Ermolenko DN, Zhang ZD, et al. Naked molerat has increased translational fidelity compared with the mouse, as well as a unique 28 S ribosomal RNA cleavage. Proc Natl Acad Sci U S A. 2013;110:17350-5.

39. Pfaffl MW. A new mathematical model for relative quantification in real-time RT-PCR. Nucleic Acids Res. 2001;29:45e-45.

40. Boda E, Pini A, Hoxha E, Parolisi R, Tempia F. Selection of Reference Genes for Quantitative Real-time RT-PCR Studies in Mouse Brain. J Mol Neuro. 2008:37:238-53.

41. Vandesompele J, De Preter K, Pattyn F, Poppe B, Van Roy N, De Paepe A, et al. Accurate normalization of real-time quantitative RT-PCR data by geometric averaging of multiple internal control genes. Genome Biol. 2002;3:RESEARCH0034.

42. Hellemans J, Vandesompele J. In: Biassoni R, Raso A, editors. Quantitative Real-Time PCR. New York: Springer New York; 2014. p. 19-26.

43. Axtner J, Sommer S. Validation of internal reference genes for quantitative real-time PCR in a non-model organism, the yellow-necked mouse. Apodemus flavicollis BMC Res Not. 2009;2:264.

44. Radonic A, Thulke S, Mackay IM, Landt O, Siegert W, Nitsche A. Guideline to reference gene selection for quantitative real-time PCR. Biochem Biophys Res Comm. 2004;313:856-62.

45. Valente V, Teixeira SA, Neder L, Okamoto OK, Oba-Shinjo SM, Marie SK, et al. Selection of suitable housekeeping genes for expression analysis in glioblastoma using quantitative RT-PCR. BMC Mol Biol. 2009;10:17.

46. Chapman JR, Waldenström J. With Reference to Reference Genes: A Systematic Review of Endogenous Controls in Gene Expression Studies. PLoS One. 2015;10, e0141853.

47. Turabelidze A, Guo S, DiPietro LA. Importance of housekeeping gene selection for accurate reverse transcription-quantitative polymerase chain reaction in a wound healing model: Accurate RT-qPCR in a wound healing model. Wound Rep Regen. 2010;18:460-6.

48. Orr ME, Garbarino VR, Salinas A, Buffenstein R. Extended Postnatal Brain Development in the Longest-Lived Rodent: Prolonged Maintenance of Neotenous Traits in the Naked Mole-Rat Brain. Frontiers in Neuroscience [Internet]. 2016; 10: 504.

49. Edrey YH, Park TJ, Kang H, Biney A, Buffenstein R. Endocrine function and neurobiology of the longest-living rodent, the naked mole-rat. Exp Gerontol. 2011:46:116-23.

50. Bruckert G, Vivien D, Docagne F, Roussel BD. Normalization of Reverse Transcription Quantitative PCR Data During Ageing in Distinct Cerebral Structures. Mol Neurobiol. 2016;53:1540-50.

51. Kouadjo KE, Nishida Y, Cadrin-Girard JF, Yoshioka M, St-Amand J. Housekeeping and tissue-specific genes in mouse tissues. BMC Genomics. 2007;8:127.

52. Fang et al., Adaptations to a subterranean environment and longevity revealed by the analysis of mole rat genomes, Cell Rep. 2014 Sep 11;8(5): 1354-64. doi:10.1016/j.celrep.2014.07.030

53. van den Bergen JA, Miles DC, Sinclair AH, Western PS. Normalizing Gene Expression Levels in Mouse Fetal Germ Cells. Biol Reprod. 2009;81:362-70.

54. Hoagland EN, Sherwood TW, Lee KG, Walker CJ, Askwith CC. Identification of a Calcium Permeable Human Acid-sensing Ion Channel 1 Transcript Variant. J Biol Chem. 2010;285:41852-62.

55. Garcia-Anoveros J, Samad TA, Zuvela-Jelaska L, Woolf CJ, Corey DP. Transport and localization of the $\mathrm{DEG} / \mathrm{ENaC}$ ion channel $\mathrm{BNaClalpha}$ to peripheral mechanosensory terminals of dorsal root ganglia neurons. J Neurosci. 2001:21:2678-86

56. Ziemann AE, Allen JE, Dahdaleh NS, Drebot II, Coryell MW, Wunsch AM, et al. The Amygdala Is a Chemosensor that Detects Carbon Dioxide and Acidosis to Elicit Fear Behavior. Cell. 2009;139:1012-21.

57. Donier E, Rugiero F, Jacob C, Wood JN. Regulation of ASIC activity by ASIC4-new insights into ASIC channel function revealed by a yeast two-hybrid assay. Eur J Neurosci. 2008:28:74-86.

58. Biagini G, Babinski K, Avoli M, Marcinkiewicz M, Seguela P. Regional and subunit-specific downregulation of acid-sensing ion channels in the pilocarpine model of epilepsy. Neurobiol Dis. 2001;8:45-58.

\section{Submit your next manuscript to BioMed Central and we will help you at every step:}

- We accept pre-submission inquiries

- Our selector tool helps you to find the most relevant journal

- We provide round the clock customer support

- Convenient online submission

- Thorough peer review

- Inclusion in PubMed and all major indexing services

- Maximum visibility for your research

Submit your manuscript at www.biomedcentral.com/submit
Biomed Central 